\title{
VALORES CRÍTICOS PARA EVALUAR PROYECTOS AGRÍCOLAS EN ESCENARIOS DE PRECIOS ESTOCÁSTICOS
}

\section{CRITICAL VALUES TO EVALUATE AGRICULTURAL PROJECTS IN SCENARIOS OF ESTOCHASTIC PRICES}

\author{
Roberto Domínguez Alonso, José de J. Brambila Paz, J. Saturnino Mora Flores* \\ y Miguel A. Martínez Damián
}

\begin{abstract}
"Programa de Economía, Instituto de Socioeconomía Estadística e Informática, Colegio de Postgraduados. Km. 36.5 Carretera México-Texcoco. 56230, Montecillo, Texcoco, Estado de México. Tel. 01(595)95-20222, Ext. 1835.

\section{RESUMEN}

A partir de la entrada en vigor del Tratado de Libre Comercio de América del Norte (TLCAN), en México ha habido una reconversión de los cultivos considerados menos rentables como algunos cereales, hacia otros más rentables como frutales y hortalizas. Al hacer la estimación del valor que indica cuántas veces mayor debe ser el valor del proyecto respecto a la inversión para que ésta sea rentable, en escenarios de precios estocásticos, dicho valor resultó mayor en frutales y hortalizas que en los considerados menos rentables. El valor crítico promedio fue de $\mathbf{1 . 3 9}$ para cereales, de $\mathbf{1 . 4 5}$ para hortalizas y de 1.64 para frutales. Por tanto, la inversión en frutales y hortalizas es más riesgosa y se debe esperar un mayor valor del proyecto que en cultivos considerados menos rentables.

Palabras clave: Inversión, valor crítico, rentabilidad.

\section{SUMMARY}

Since the entry into the North American Free Trade Agreement (NAFTA), in México there has been a conversion of those crops considered less profitable, such as cereals, to others more profitable such as fruits and vegetables. However, on carrying out an estimate of the value that indicates how many times greater should be the value of the project regarding the investment, in order to be profitable under stochastic prices scenarios, it was noted that this value is greater for fruits and vegetables than for those crops considered less profitable. The critical value was 1.39 for cereals, $\mathbf{1 . 4 5}$ for vegetables, and $\mathbf{1 . 6 4}$ for fruits. Therefore, the investment in the production of fruit and vegetables is riskier, and should have a higher value project that in the case of less profitable crops.

Index words: Investment, critical values, profitability.

\section{INTRODUCCIÓN}

A partir de la entrada en vigor del Tratado de Libre Comercio de América del Norte (TLCAN), en México se ha presentado una reconversión de los cultivos considerados menos rentables, como los de cereales, a otros de mayor rentabilidad como frutales y hortalizas (Quintana, 2003; González, 2002). La mayor rentabilidad de los frutales y hortalizas con respecto a cereales ha sido el principal argumento para apoyar el cambio de estos últimos, al cultivo de los primeros (Martínez y Díaz, 2005).

De acuerdo con datos del Sistema de Información Agroalimentaria de Consulta (SIACON), de la superficie nacional dedicada a cultivos agrícolas, en promedio 43.5 $\%$ se destinó a la producción de cereales durante el periodo de 1994 a 2006, mientras que el cultivo de frutas y hortalizas ocuparon una superficie de $5.8 \%$ y $2.6 \%$, respectivamente. En contraste, las frutas aportaron $18 \%$ del valor total agrícola y las hortalizas $16 \%$, mientras que los cereales aportaron $21.9 \%$ de dicho valor para el mismo periodo. A pesar de que en México se sigue destinando la mayor parte de la superficie al cultivo de cereales, ésta ha presentado una disminución significativa al pasar de 10363661 ha y de representar $49.3 \%$ de la superficie total en 1994, a ocupar $40.3 \%$ con 8630588 ha en 2006.

Dentro del grupo de hortalizas los productos de mayor importancia en la superficie cultivada y por el valor que generan, son: calabacita, cebolla, chile verde, pepino, tomate, tomate de cáscara y zanahoria. Estos siete productos en el año 2006 ocuparon $56 \%$ de la superficie sembrada de hortalizas y generaron $70 \%$ del valor total aportado por las hortalizas. En el grupo de frutales los de mayor importancia son: naranja, plátano, aguacate, limón, guayaba, mango y manzana, que en el 2006 generaron $59 \%$ del valor generado y representaron $69 \%$ de la superficie total sembrada de frutales. 
El objetivo de esta investigación fue encontrar un valor crítico (valor mínimo) de un proyecto de inversión para tres grupos de productos (cereales, frutas y hortalizas), en presencia de volatilidad de precios, que considere el riesgo e incertidumbre de invertir en la producción de estos cultivos. Al respecto, se postula que el valor crítico de los cereales debe ser menor que el de frutas y hortalizas.

\section{MATERIALES Y MÉTODO}

Para calcular el valor actual de un proyecto que tiene flujo de efectivo constante, lo que tradicionalmente se hace es descontar el flujo de efectivo de varios años y calcular la suma de los valores resultantes:

$$
V=\frac{C}{(1+i)}+\frac{C}{(1+i)^{2}}+\frac{C}{(1+i)^{3}}+\ldots \ldots \ldots \ldots
$$

Donde: $V=$ valor actual del proyecto; $C=$ pago periódico y constante; $i=$ tasa de interés de mercado.

Este cálculo puede ser tedioso cuando el número de periodos es grande. No obstante, se puede simplificar debido a que las fracciones de la expresión anterior forman una progresión geométrica, porque cada una es igual al anterior multiplicado por $\frac{1}{(1+i)}$; por tanto, el

valor actual de este tipo de proyectos puede estimarse como la suma de los términos de una progresión geométrica:

$$
\mathrm{Si}: \frac{C}{(1+i)}=a \quad \mathrm{y} \quad \frac{1}{(1+i)}=x
$$

Donde: $a=$ primer término de la progresión; $x=$ razón de la progresión.

Entonces: $V=a\left(1+x+x^{2}+x^{3}+\ldots \ldots \ldots \ldots x^{n-1}\right)$

Al multiplicar ambos lados de la ecuación por $x$ se tiene: $V x=a\left(x+x^{2}+x^{3}+\ldots \ldots \ldots \ldots \ldots \ldots x^{n}\right)$

Si a $V$ se le resta $V x$, resulta: $\quad V-V x=a-a x^{n}$

$$
\text { Entonces: } \quad V=\frac{a\left(1-x^{n)}\right.}{(1-x)}=C\left[\frac{1-(1+i)^{-n}}{i}\right]
$$

La Ec. 1 es la que se utiliza para obtener la suma de $n$ términos consecutivos de un proyecto con sólo saber el primer término a sumar, la razón y el número de términos ${ }^{1}$, lo cual tiene como supuesto que el flujo de efectivo es constante y seguro. Si el valor del proyecto tiene un comportamiento estocástico debido a que los precios del producto final tienen un comportamiento aleatorio, entonces se deberá encontrar un valor crítico del proyecto de inversión para conservar el valor presente.

La oportunidad de invertir en un proyecto que tiene un comportamiento estocástico es similar a una opción de compra. Una opción de compra otorga al titular, previo pago de una prima, el derecho más no la obligación de adquirir un activo, a un precio preestablecido, durante un tiempo determinado. Cuando un inversionista efectúa un gasto de inversión irreversible, ejerce o deja que expire su opción para invertir. Esta opción de invertir proporciona la posibilidad de esperar a que llegue nueva información que afectará la conveniencia o el momento de invertir; si la inversión se realiza y las condiciones de mercado cambian adversamente habrá costos que no podrán ser recuperados, pérdida de valor que es un costo de oportunidad que debe ser incluido como parte de los costos de inversión. Como resultado de lo anterior, la regla simple del valor actual neto (VAN), que señala que se debe invertir si el flujo de fondos actualizados es por lo menos igual al flujo de costos actualizados, debe ser modificada. El valor de los beneficios debe ser mayor al de los costos, en una cantidad igual al valor de mantener la opción de inversión abierta.

La regla del VAN positivo debe modificarse como lo proponen Dixit y Pindyck (1994), quienes hacen un análisis al tomar como base el modelo de Mc Donald y Siegel (1986), en el que se considera el problema del momento en que será óptimo realizar una inversión irreversible $I$, cuyo valor $V$ evoluciona de acuerdo con el siguiente proceso estocástico browniano²:

$$
d V=\alpha V d t+\sigma V d z \quad(\text { Ec. 2) }
$$

\footnotetext{
${ }^{1}$ Como caso particular, puede calcularse el valor actual de un proyecto cuando tiene flujos de fondos constantes y perpetuos $(n \rightarrow \infty)$ con la expresión: $\frac{C}{i}$, donde $\mathrm{i}=$ tasa de interés, y $C=$ flujo de fondos constantes.

${ }^{2}$ También llamado proceso de Wiener, es un proceso estocástico de tiempo continuo con tres propiedades importantes. Primero es un proceso de Markov, lo que significa que la probabilidad de distribución para todos los valores futuros del proceso sólo depende de su valor actual, y no es afectada por los valores pasados o por cualquier otra información actual. Segundo, tiene incrementos independientes. Y tercero, los cambios en el proceso sobre cualquier intervalo de tiempo finito son distribuidos normalmente. Ver Dixit y Pindyck (1994).
} 
Donde: $d V=$ movimiento del valor del proyecto; $\alpha=$ tasa de crecimiento del proyecto; $V=$ valor del proyecto; $\sigma=$ desviación estándar del valor del proyecto; $d z=$ incremento de un proceso de Wiener.

La Ec. 2 implica que el valor actual del proyecto es conocido, pero los valores logarítmicos se distribuyen normalmente con varianza proporcional al tiempo. Así, no obstante que la información llega a través del tiempo (el inversionista observa el cambio en el valor $V$ ), el valor futuro del proyecto es siempre incierto.

Lo que se desea es tener una regla que maximice el valor de una oportunidad de inversión, la cual se denota por $\mathrm{F}(V)$. Dado que el valor de invertir en $\mathrm{t}$ es igual a $(V t-I)$, el objetivo es maximizar su valor presente:

$$
F(V)=\max E\left[\left(V_{T}-I\right) e^{-\rho T}\right]
$$

Donde: $T=$ momento futuro (desconocido) en el que la inversión es realizada; $\rho=$ tasa de descuento relevante y la maximización se encuentra sujeta a la dinámica de la Ec. 2. Para que el problema tenga sentido, se asume que $\alpha<\rho$; de otra manera no se invertiría, y $\mathrm{F}(V)$ se volvería infinito. Se denota $\delta$ a la diferencia $\rho-\alpha$; en consecuencia se asume que $\delta>0$.

La solución a este problema es desarrollada por Dixit y Pindick (1994). La regla de inversión óptima toma la forma de un valor crítico $V^{*}$, que es el óptimo a invertir una vez que $V \geq V^{*}$. El valor de una oportunidad de inversión (si se supone que la empresa invierte sólo cuando $V$ iguala a $V^{*}$ ) es:

$$
F(V)=\mathrm{A} V^{\beta} \quad(\text { Ec. } 4)
$$

Donde:

$\beta=\frac{1}{2}-\frac{\rho-\delta}{\sigma^{2}}+\sqrt{\left(\frac{\rho-\delta}{\sigma^{2}}-\frac{1}{2}\right)^{2}+2 \frac{\rho}{\sigma^{2}}}>1$

El valor de $V^{*}$ esta dado por:

$$
\begin{aligned}
V^{*} & =\frac{\beta}{(\beta-1)} I \\
\mathrm{Y} \quad A & =\frac{\left(V^{*}-I\right)}{\left(V^{*}\right)^{\beta}}=\frac{(\beta-1)^{\beta-1}}{\beta^{\beta} I^{\beta-1}}
\end{aligned}
$$

El punto importante es que si $\beta>1$, entonces $V *>$ $I$. Así, la incertidumbre y la irreversibilidad abren una brecha entre el valor crítico $V *$ y el costo de la inversión I.

Con las Ecs. 5 y 6 se puede calcular el valor crítico de un proyecto $\left(V^{*}\right)$ que indique cuántas veces mayor tiene que ser el valor del proyecto a la inversión para que no haya pérdidas. Para ello se necesita saber la tasa de descuento $(\rho)$ y calcular la media de la tasa de crecimiento de los precios $(\alpha)$ y su varianza $\left(\sigma^{2}\right)$.

La información de los índices de precios de los diferentes cultivos considerados por grupo, así como el índice general de precios al consumidor se obtuvieron de las estadísticas del Banco de México (Banxico), para el periodo de enero de 1994 a diciembre de 2007.

Para calcular el valor crítico primeramente se obtuvo el comportamiento de los precios de 21 productos agrícolas (siete frutales, siete hortalizas y siete cereales), se utilizó el índice de precios al productor de cada cultivo y se deflactaron con el índice general de precios al consumidor, base diciembre de 2003. Posteriormente se calculó la tasa de crecimiento continua de los precios, que es el logaritmo natural del cociente del año $t$ entre el año anterior $(t-1)$. Con estas tasas de crecimiento se calculó la media y la varianza de esos productos, y una vez obtenidas para cada cultivo se calculó el valor crítico con una tasa de $10 \%$, al considerar los siguientes valores:

inversión $I=1$; tasa de descuento $\rho=0.10$; tasa de descuento menos la media de la tasa de crecimiento de los precios $\delta=(\rho-\alpha)$; y varianza de la tasa de crecimiento de los precios $\left(\sigma^{2}\right)$.

Para obtener el valor de $\beta$ se utilizan los valores anteriores y la Ec. 5. Por ejemplo, para el caso de aguacate se tiene: $I=1 ; \rho=0.10 ; \alpha=0.027 ; \sigma^{2}=$ 0.1377 .

Al sustituir en la Ec. 5:

$$
\beta=\frac{1}{2}-\frac{0.10-(0.10-0.0274)}{0.1377}
$$

$$
+\sqrt{\left(\frac{0.10-(0.10-0.0274)}{0.1377}-\frac{1}{2}\right)^{2}+2 \frac{0.10}{0.1377}}=1.544
$$

Al sustituir el valor de $\beta$ en la Ec. 6 el valor crítico del proyecto será:

$$
V^{*}=\frac{1.544}{(1.544-1)} I=2.8397
$$


Lo anterior significa que en escenarios de precios estocásticos sólo se debe invertir si el valor del proyecto es cuando menos 2.83 veces mayor a la inversión.

\section{RESULTADOS Y DISCUSIÓN}

Los resultados que aquí son presentados corresponden al valor crítico de los 21 productos, y en promedio los valores críticos fueron de 1.39 para cereales, de 1.45 para hortalizas y de 1.64 para los frutales (Cuadro 1). Lo anterior muestra que la decisión de invertir en un proyecto para producir cereales, tiene menos riesgo que la inversión en proyectos de producción de hortalizas, y estos proyectos son aún menos riesgosos que sus correspondientes en frutales. Esto concuerda con los resultados de Martínez y Díaz (2005), quienes encontraron el mismo grado de riesgo para los mismos grupos de cultivos.

Cuadro 1. Valor crítico promedio, valor crítico máximo y valor crítico mínimo de tres grupos de cultivos.

\begin{tabular}{lccc}
\hline Valor crítico & Frutales & Hortalizas & Cereales \\
\hline Promedio & 1.64 & 1.45 & 1.39 \\
V. máximo & 2.83 & 1.73 & 1.74 \\
V. mínimo & 1.34 & 1.21 & 1.24 \\
\hline
\end{tabular}

Además que la afirmación por grupos es sostenible, también es posible analizar a cada cultivo en particular. Los 21 productos se clasificaron en grupos de riesgo (bajo, medio y alto), de acuerdo con la dimensión del valor crítico. En el grupo de riesgo bajo se ubicaron a dos cereales, (arroz y sorgo) y dos hortalizas (tomate y tomate de cáscara) (Cuadro 2). Como se observa, por grupos de productos los proyectos de inversión en algunos cereales y hortalizas presentan menor riesgo.

Dado que los valores mínimos y máximos de cada grupo son cercanos (Cuadro 1), existe la posibilidad de que en lo individual se obtengan comportamientos de riesgo similar sin importar el tipo de cultivo. El Cuadro 2 presenta los cultivos de bajo riesgo, cuyos valores críticos fueron inferiores a 1.3. Si bien el tomate aparece como cultivo de bajo riesgo, es de resaltar que la fuente de incertidumbre considerada es el precio, y otras fuentes alternativas de riesgo como clima, plagas y otros factores de mercados, no fueron considerados. Es de destacar que en este grupo de bajo riesgo no aparece frutal alguno de los aquí estudiados. En el trabajo desarrollado por Martínez y Díaz (2005) no se hicieron comparaciones por cultivo, y por ello no se pueden contrastar con los aquí obtenidos.
Cuadro 2. Valores críticos de $\beta$ y de $V^{*}$, del grupo de productos con riesgo bajo.

\begin{tabular}{lcc}
\hline Producto & $\beta$ & $V^{*}$ \\
\hline Tomate & 5.720 & 1.2119 \\
Arroz & 5.075 & 1.2454 \\
Tomate de cáscara & 4.933 & 1.2543 \\
Sorgo & 4.454 & 1.2895 \\
\hline
\end{tabular}

En el grupo de riesgo medio se ubicaron cinco frutales: plátano, manzana, mango, guayaba y naranja, con valores críticos de 1.3481 a 1.4947; cuatro cereales: trigo, cebada maíz y frijol, con valores de 1.3132 a 1.4202 ; y tres hortalizas: chile fresco, calabacita y pepino, con valores críticos de 1.3219 a 1.4947 (Cuadro 3).

Cuadro 3. Valores críticos de $\beta$ y de $V^{*}$, del grupo de productos con riesgo medio.

\begin{tabular}{lcc}
\hline Producto & $\beta$ & $V^{*}$ \\
\hline Trigo & 4.193 & 1.3132 \\
Chile fresco & 4.106 & 1.3219 \\
Cebada & 3.939 & 1.3402 \\
Plátano & 3.873 & 1.3481 \\
Manzana & 3.843 & 1.3517 \\
Mango & 3.782 & 1.3595 \\
Maíz & 3.504 & 1.3994 \\
Frijol & 3.379 & 1.4204 \\
Guayaba & 3.169 & 1.4611 \\
Calabacita & 3.153 & 1.4645 \\
Pepino & 3.077 & 1.4815 \\
Naranja & 3.021 & 1.4947 \\
\hline
\end{tabular}

Los cultivos de riesgo medio, cuyos valores críticos mínimos y máximos (Cuadro 3) explican que provengan de los tres grupos considerados, contrastan con los de cultivos de mayor riesgo (Cuadro 4) en los que destaca la preponderancia de hortalizas y frutales. En el grupo de riesgo alto se ubicaron dos hortalizas: zanahoria y cebolla; dos frutales: limón y aguacate (éste último fue el de mayor valor crítico de los 24 cultivos, con 2.8397); y un cereal, garbanzo (Cuadro 4).

Cuadro 4. Valores críticos de $\beta$ y de $V^{*}$, del grupo de productos con riesgo alto.

\begin{tabular}{lcc}
\hline Producto & $b$ & $V^{*}$ \\
\hline Limón & 2.514 & 1.6603 \\
Zanahoria & 2.399 & 1.7148 \\
Cebolla & 2.353 & 1.7389 \\
Garbanzo & 2.339 & 1.7468 \\
Aguacate & 1.544 & 2.8397 \\
\hline
\end{tabular}

Del análisis tanto por grupos de cultivos como por cultivos individuales se desprende que en general el valor crítico promedio es menor para cereales, seguido por hortalizas y frutales; pero cuando se requiere tomar una decisión de inversión, también es necesario considerar información individual por cultivo, debido a que hay cereales riesgosos y frutales de bajo riesgo. 


\section{CONCLUSIONES}

En promedio, el valor crítico de los cereales es menor al de los frutales y hortalizas, lo cual apoya la hipótesis planteada en esta investigación; la inversión en la producción de frutales y hortalizas es más riesgosa que con cereales.

Aún cuando se pueda plantear la conveniencia de realizar una reconversión en el agro mexicano en favor de los frutales y hortalizas, dada su mayor rentabilidad, no se debe ignorar que no basta con tener un mayor valor del proyecto, sino que también se debe tomar en cuenta qué tan grande debe de ser ese valor para evitar pérdidas. Además, debe tomarse en cuenta el riesgo específico para cada clase de cultivo, independiente del grupo al que pertenece.

\section{BIBLIOGRAFÍA}

Banco de México (1994 a 2007) Estadísticas de índices de precios al consumidor, general y por producto en México. Boletines mensuales. Consultado en línea: www.banxico.org.mx. (Junio 2008).

Díaz C M, T R Juárez, C M Gómez (2007) Conformación de una cartera de inversión óptima de cultivos agrícolas para México. Econ. Soc. Territ. 7:49-63.

Dixit A, R Pindyck (1994) Investment Under Uncertainty. Princeton University Press, Princeton, USA. 468 p.

González E A (2002) Dinámica de los Cultivos Básicos en la Liberación Comercial de México: Un Modelo Dinámico Multisectorial de Equilibrio General. Libro Técnico 5. División Agrícola INIFAP. Chapingo, México. 138 p.

Martínez D M, C M Díaz (2005) Comparación de la varianza estimada de los índices de precios al consumo de cereales, frutales y hortalizas en México, 1980-2002. Anál. Econ. 20:75-88.

Mc Donald R, D Siegel (1986) The value of waiting to Invest. Quart. J. Econ. 101:707-728.

Quintana S V (2003) El circulo vicioso de del Tratado de Libre Comercio de América del Norte: la amarga experiencia mexicana en el agro a partir del TLCAN. Deslinde 33:26-38.

Secretaría de Agricultura, Ganadería, Desarrollo Rural, Pesca y Alimentación (2003) Sistema de Información Agropecuaria de Consulta (SIACON). Versión 3.2. Centro de Estadística Agropecuaria, México. Consultado en línea: www.oeidrustamaulipas.gob.mx/cd_anuario_07/siacon19802007wv.html. (Junio de 2008) 